\title{
Lutetium Lu 177 Dotatate
}

National Cancer Institute

\section{Source}

National Cancer Institute. Lutetium Lu 177 Dotatate. NCI Thesaurus. Code C95020.

A radioconjug ate consisting of the tyrosine-containing somatostatin analog Tyr3octreotate (TATE) conjug ated with the bifunctional, macrocyclic chelating agent tetraazacyclododecanetetra-acetic acid (DOTA) and radiolabeled with the beta-emitting radioisotope lutetium Lu 177, with potential imaging and antineoplastic activities. Lutetium Lu 177 dotatate binds to somatostatin receptors (SSTRs), with high affinity to type 2 SSTR, present on the cell membranes of many types of neuroendocrine tumor (NET) cells. Upon binding and internalization, this radioconjug ate specifically delivers a cytotoxic dose of beta radiation to SSTR-positive cells. Tyr3-octreotate (TATE) is an octreotide derivative in which phenylalanine at position 3 is substituted by tyrosine and position 8 threoninol is replaced with threonine. SST Rs have been shown to be present in large numbers on NET and their metastases, while most other normal tissues express low levels of SSTRs. 